\title{
Management of primary antibody deficiency by consultant immunologists in the United Kingdom: a paradigm for other rare diseases
}

\author{
G P Spickett, T Askew, H M Chapel
}

\begin{abstract}
Variation in clinical practice and its effect on outcome is little known for rare diseases such as primary antibody deficiency. As part of a national audit a survey of all 30 consultant immunologists in the United Kingdom dealing with primary antibody deficiency syndromes in adults and children was carried out in 1993 to ascertain their practices in diagnosis and management. Consensus guidelines were published after the survey was completed. Comparison of the survey results of clinical practice at the time the guidelines were published with the standards identified highlighted that the practice of a minority of specialists was at variance with their peers and with the consensus document, particularly in the use of intramuscular immunoglobulin, the dose and frequency of intravenous immunoglobulin, and target trough immunoglobulin $G$ concentration, which has implications for the quality of patient care. However, much closer agreement existed in the key areas of management, such as diagnosis and selection of intravenous immunoglobulin. The approach and the problems identified are relevant to the management of other rare diseases, in which diagnosis and management is complex and there are few specialists with the necessary knowledge to undertake such care. This survey, the first attempted audit of practice, shows that within a motivated group of specialists highly significant differences in practice may exist, and the authors emphasise the importance of setting clear guidelines against which care can be assessed.

(Quality in Health Care 1995;4:263-268)
\end{abstract}

Keywords: primary antibody deficiency, guidelines, audit

\section{Introduction}

Little information exists on how quality of specialist services for patients with rare diseases can be assessed and how variations in that care offered may affect clinical outcome.

Not all patients are managed by consultants with a special interest in primary immunodeficiencies; some patients are managed by paediatricians, haematologists, and other
Primary antibody deficiency syndromes are rare disorders leading to the failure to produce adequate levels of protective antibodies. ${ }^{1}$ Although some are inherited, such as X linked agammaglobulinaemia, and present early in childhood, others are of uncertain aetiology and can present at any age, such as common variable immunodeficiency. The clinical presentation is usually with recurrent bacterial infections, although autoimmune disease is common in common variable immunodeficiency. ${ }^{2}$ Most patients will present in the first instance to their general practitioner, who must be alert to the possibility and seek an appropriate second opinion. Treatment is by giving replacement immunoglobulin to compensate for the missing antibodies, using immunoglobulin $G$ prepared from large donor pools and administered by the intramuscular or, more commonly, the intravenous route. Antibiotics and plasma are ineffective alone. Failure to treat adequately leads to progressive structural damage, particularly of sinuses and lungs from recurrent infections ${ }^{3}$ and to a significant risk of serious invasive infection and early mortality (G P Spickett and T Askew, in preparation). In the United Kingdom these conditions are considerably underdiagnosed compared with Scandinavia. Furthermore, small studies have shown a considerable diagnostic delay - on average about seven years from onset of symptoms to diagnosis (G P Spickett and $T$ Askew, in preparation) - leading to needless additional morbidity and mortality. The reasons for this are not certain but are likely to reflect ignorance among medical practitioners of the existence of these rare conditions.

physicians. Published work gives no clear guidance on the optimum management of such patients, other than to point to certain minimum standards. A medical panel convened by the Primary Immunodeficiency Association produced a consensus document in 1994 on the management of such disorders, ${ }^{5}$ which is summarised in the following box.

As part of a national audit of the quality of care received by patients with antibody deficiency, it was thought to be important to ascertain the views of the immunologists managing such patients and to compare existing practice with the standards in the consensus document. If specialists with a particular interest in these conditions are not agreed on the optimum approach then it will be difficult to convince non-specialists of the correct management approach. The problems 
Major recommendations of consensus document on management of primary antibody deficiency

\section{All patients should be referred to an} immunologist for precise diagnosis and long term management, often in conjunction with an appropriate specialist or paediatrician, or both

2 The following investigations should be carried out: serum immunoglobulins, in relation to normal range for age, existing immunoglobulin $\mathrm{G}$ responses to previous infections and immunisations, test immunisation with protein and polysaccharide antigens (immunoglobulin $\mathrm{G}$ ), immunoglobulin $\mathrm{G}$ subclasses related to normal ranges for age and lymphocyte subpopulations. Long term tissue damage should be documented and investigated before treatment

3 Patients are likely to require doses of intravenous immunoglobulin between $0 \cdot 4-1 \mathrm{~g} / \mathrm{kg} / \mathrm{month}$, given in divided doses, usually two weeks apart, since the half life of immunoglobulin $\mathrm{G}$ is three weeks. Intramuscular immunoglobulin is now largely superseded

4 Patients should be considered for home treatment programmes; those unsuitable should have their infusions in their local hospital supervised by a nurse specialising in immunology

5 All patients should be reviewed every 3-6 months by a consultant immunologist for adequacy of treatment and development of complications

6 Patients should be made aware of the Primary Immunodeficiency Association and invited to receive relevant literature

7 The consensus document also lists recognised centres for specialist diagnosis and management and those centres providing approved training for home treatment.

of the quality of care in such a group of life threatening rare diseases are applicable to other rare diseases, where access to specialist expertise is limited. The first problem for patients with a rare disease is correct diagnosis, either in primary or secondary care: for patients with antibody deficiency diagnosis is often long delayed. ${ }^{4}$ If patients get as far as specialist centres, what safeguards are there that they will receive optimum care? There are difficulties in organising audit among a group of specialists distributed the length and breadth of the United Kingdom, more so when published reports provide no clear guidance on absolute standards and those standards that are available are set by those involved in the audit.

In 1993 we undertook a questionnaire survey of consultant immunologists to investigate the level of agreement on the correct approach to managing patients with a defined group of rare immunodeficiency disorders and to assess their quality of care. This approach may have implications for the investigation of the care of other rare disorders. In order to improve standards of care the participants were sent the results of the survey.

\section{Subjects and methods}

We sent a questionnaire to all consultant immunologists thought to be involved in the care of patients with primary antibody deficiency. The selection was based on our personal knowledge of the interests and workload of the consultants; those who did not specialise in the care of patients with antibody deficiency were not circulated. The survey was not anonymous. The questionnaire contained specific questions about diagnosis and management of antibody deficiency syndromes, and respondents were asked to tick boxes to indicate their views to specific questions. The box shows an abbreviated form of the questions.

\section{Information sought in questionnaire}

1 Do you have direct responsibility for the care of patients with primary antibody deficiency?

2 If no, do you advise other clinicians on managing patients with primary antibody deficiency?

3 For a newly presenting adult patient with suspected antibody deficiency which investigations would you request on all patients (measurement of immunoglobulins, immunoglobulin $\mathrm{G}$ subclasses, specific antibacterial and antiviral antibodies, lymphocyte surface markers)?

4 If the diagnosis is compatible with common variable immunodeficiency would you: use intramuscular immunoglobulin, intravenous immunoglobulin, either, or accept patient's choice?

5 For those using intramuscular immunoglobulin dose and frequency was requested.

5a Reasons for preferring intramuscular immunoglobulin over intravenous immunoglobulin

$5 b$ Information on intramuscular immunoglobulin product used

6 Information on method of selection of an intravenous immunoglobulin product for a patient

7 Do you believe that changes in intravenous immunoglobulin product are potentially hazardous/undesirable?

8 Which products do you currently use (list of available products given)?

9 Respondents were asked to rank 11 criteria used in the selection of an intravenous immunoglobulin product, see table

10 Were respondents consulted over purchasing contracts for intravenous immunoglobulin, whether this adversely affected clinical management, and effect of changes in contracts on prescribing?

11 Details of dose of intravenous immunoglobulin used, method of calculating dose, and target trough immunoglobulin $\mathrm{G}$

12 Frequency of intravenous immunoglobulin administration - either fixed (with interval) or variable

13 Investigations carried out to assess adequacy of treatment 
14 If the patient was a child with $\mathrm{X}$ linked agammaglobulinaemia what treatment would you recommend (choice of intramuscular immunoglobulin, intravenous immunoglobulin, or either depending on patient)?

15 If you had found an immunoglobulin G subclass/specific antibody deficiency would you: use continuous antibiotics, intramuscular immunoglobulin, intravenous immunoglobulin, not treat at all?

16 For patients with good venous access and appropriate circumstances do you always offer the option of home treatment? Those answering "no" were asked for reasons why not

17 For newly diagnosed patients, do you provide them with printed information about their condition/treatment to take away? Those answering "no" were asked for reasons

18 Do you routinely give patients details of patient society (Primary Immunodeficiency Association)? Those answering "no" were asked for reasons

19 How often do you review adult patients with antibody deficiency?

The questionnaire data were entered on to a computer database for analysis. In view of the small number of respondents, the analysis was carried out with simple descriptive statistics to evaluate the views of the group. The results were compared against the standards identified in the consensus document.

\section{Results}

In all, 30 questionnaires (100\% response rate) were received from immunologists actively managing patients with rare diseases, although some prompting was required in two cases.

CONSULTANTS' RESPONSIBILITIES

Of the 30 respondents, 23 had direct responsibility for patients' care, seven of whom also

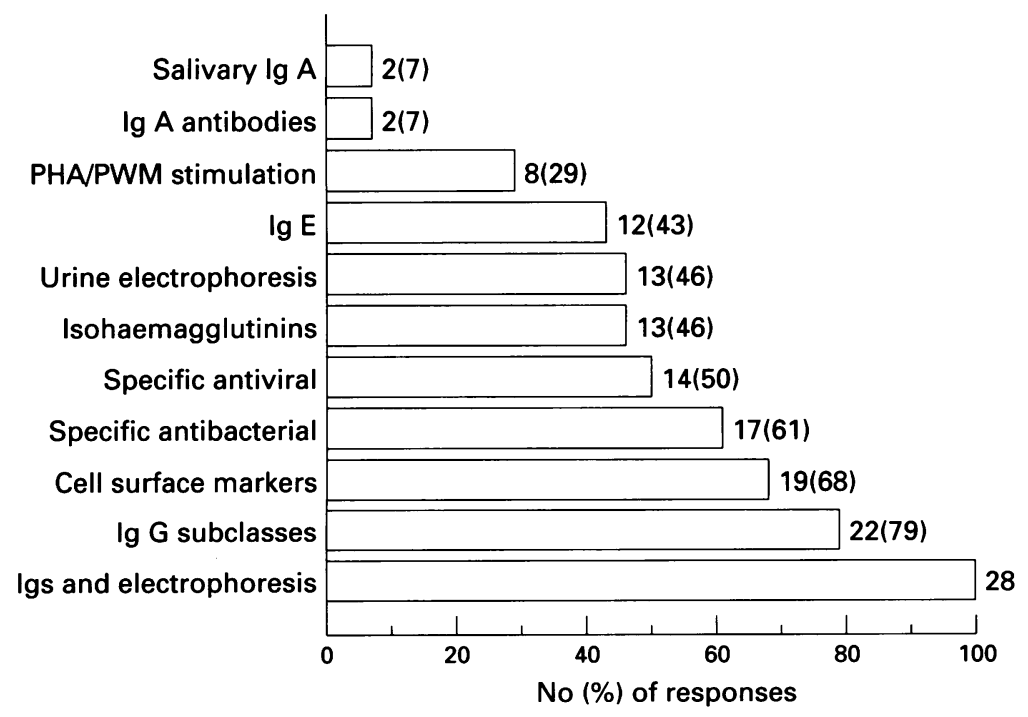

Responses of 28 consultant immunologists answering question on investigations requested for newly presenting adult patient with suspected antibody deficiency. Consensus standard recommended for all patients is for measurement of immunoglobulins, immunoglobulin $G$ subclasses, specific antibacterial and antiviral antibodies, and lymphocyte surface markers (Ig = immunoglobulin, $P H A=$ phytohaemagglutinin, $P W M=$ pokeweed mitogen) advised other clinicians on management; five respondents had no direct responsibility, four of whom advised other clinicians on management; and two gave no response. This means that some patients are not receiving regular review directly by a consultant immunologist, which is a standard of the consensus.

\section{DIAGNOSTIC TESTS}

Respondents were asked to indicate which investigations they would carry out on all patients with a suspected diagnosis of antibody deficiency. The figure shows the results for the 28 respondents who had direct or indirect responsibility for patients. Although all consultants would measure immunoglobulins, other investigations were not always thought necessary. Only 19 measured lymphocyte surface markers, 17 specific antibacterial antibodies, and 14 antiviral antibodies, all of which are recommended in the consensus document. Some investigations listed may clearly be viewed as second line and carried out only in particular clinical instances.

TREATMENT OF PATIENTS WITH COMMON VARIABLE IMMUNODEFICIENCY, $X$ LINKED AGAMMAGLOBULINAEMIA, IMMUNOGLOBULIN G SUBCLASS OR SPECIFIC ANTIBODY DEFICIENCY WITH INFECTIONS

In a patient with common variable immunodeficiency 20 immunologists responded that they would immediately recommend the use of intravenous immunoglobulin whereas two would use either intramuscular immunoglobulin or intravenous immunoglobulin and six would leave the choice to patients. Immunologists using intramuscular immunoglobulin would all use a loading schedule of either daily intramuscular immunoglobulin or a single intravenous dose. Despite there being three intramuscular immunoglobulin products on the market licensed for use in antibody deficiency and their uniformity the six users of intramuscular immunoglobulin would use only one type. Of major concern is the finding that four out of 28 respondents would be prepared to use intramuscular immunoglobulin if the patient was a child with $\mathrm{X}$ linked agammaglobulinaemia. General reasons given for choosing intramuscular immunoglobulin included its cost and quick administration. There was little agreement among the respondents on the optimum management of patients with symptomatic immunoglobulin $G$ subclass or specific antibody deficiency: 11 would use intravenous immunoglobulin, one intramuscular immunoglobulin, and 12 continuous antibiotics. One would use no treatment at all.

\section{HOME TREATMENT}

Eight respondents did not offer their patients the option of home treatment, even though there are considerable advantages to suitable patients. In four cases this was owing to a lack of local facilities, even though other centres are prepared to take on distant patients and many patients in home treatment programmes 
live outside the immediate catchment areas of the training centre. One respondent admitted that he had not thought about home treatment. None thought that the concept was unsafe.

\section{PATIENT INFORMATION}

Eight immunologists did not provide their patients with local information: three had not thought about it, two had no material, and two thought that it would increase anxiety. Only three immunologists did not provide their patients with information about the national patient society, the Primary Immunodeficiency Association; one was opposed to the society on the grounds that it increased anxiety.

\section{FOLLOW UP}

Eight immunologists would leave the follow up entirely to another hospital specialist and one to the general practitioner. The remainder would either follow up patients at the time of their infusions or in the outpatient department. Nineteen respondents chose a follow up interval of 3-6 monthly and only one used an interval of more than 6 months.

\section{SELECTION OF INTRAVENOUS}

IMMUNOGLOBULIN TREATMENT

At the time of the survey seven intravenous immunoglobulin preparations were available. Fifteen immunologists chose the intravenous immunoglobulin product for a specific patient, whereas eight used whatever was stocked in the hospital pharmacy. Eight immunologists believed that the available products were not appreciably different, 23 believed that changes of product in patients whose treatment was established were undesirable, and three denied that this was significant. Most respondents used a variety of immunoglobulin products.

To drive down drug costs, many regions request tenders for the supply of intravenous immunoglobulin under regional contract. Nine immunologists were consulted about the regional contract, but seven were not: it is unacceptable that those with specialist knowledge of the products involved are excluded from contributing to the discussion. Two of those not consulted thought that the unilateral imposition of a regional contract for intravenous immunoglobulin was detrimental to their clinical service. In view of the unanimity on the undesirability of changing a patient's intravenous immunoglobulin product, it is reassuring that no immunologists were obliged to change products when the regional contract changes from year to year, although 16 had had specifically to insist on continuity of supplies.

DOSAGE AND FREQUENCY OF INTRAVENOUS IMMUNOGLOBULIN TREATMENT

The consensus document recommends that the dose of intravenous immunoglobulin should be related to the patient's weight. One immunologist used a fixed dose irrespective of weight; 14 used a fixed dose determined by weight whereas 20 used a variable dose determined by the trough immunoglobulin $G$ concentration. Eight immunologists used a per infusion dose of $0 \cdot 2-0.4 \mathrm{~g} / \mathrm{kg} /$ infusion whereas seven used doses within the range $0 \cdot 2-0 \cdot 6$ $\mathrm{g} / \mathrm{kg} /$ infusion. There was very wide variation of the target trough immunoglobulin $\mathrm{G}$ concentration sought by those immunologists using this measure (range $<4 \mathrm{~g} / \mathrm{l}$ to $>8 \mathrm{~g} / \mathrm{l}$ (normal range for serum immunoglobulin $G$ about 6-16 g/l)): some respondents specified more than one target level, presumably indicating that they set the target according to clinical status. It is of concern that six were using target immunoglobulin $\mathrm{G}$ concentrations $<5 \mathrm{~g} / \mathrm{l}$, the desirable threshold in one published study. One immunologist viewed a trough level of $<4 \mathrm{~g} / \mathrm{l}$ as satisfactory despite this being less than $66 \%$ of the lower limit of normal. Three immunologists were using follow up intervals of four weeks or greater. Monitoring of treatment by trough immunoglobulin $G$ concentrations was carried out by 25 immunologists, but only 17 regularly measured lung function, a useful marker of the overall adequacy of replacement; 16 immunologists asked patients to keep symptom diaries.

\section{FEEDBACK}

All participants were sent an anonymised copy of the analysis of the results, together with a summary of the areas of particular concern, to try and improve their quality of care.

\section{Discussion}

This survey highlights the differences in approach to the management of rare group of conditions undertaken by a group of interested specialists and compares it with management standards identified in a multidisciplinary consensus. Although overall there was good agreement with the standards identified, a minority of specialists were offering care that deviated significantly from the agreed standards in a way that may prejudice the quality of care - for instance, by inappropriate use of intramuscular immunoglobulin, unduly low target concentration of immunoglobulin for treatment, and lack of specialist follow up. Patients will also be inconvenienced by lack of access to facilities for self treatment at home, lack of supportive information and information about a patient support society.

A problem with postal surveys is that they analyse "reported practice," which may differ from actual practice. However, all the participants in this survey agreed to participate in an extensive project to obtain clinical and outcome information on patients with antibody deficiency. That project is nearing completion and will provide information, by centre, of the quality of patient care. The project involves a review of the case notes of all identified patients with antibody deficiency and is being carried out in conjunction with efforts to produce an up to date register of patients with all types of immune deficiencies ( $G P$ Spickett and $T$ Askew, unpublished). An interim analysis carried out on 392 records shows that significant numbers of patients have chronic 
complications, particularly bronchiectasis and sinusitis. Complications continue to develop after referral to immunologists. It also confirms that the delay from onset of clear symptoms to referral to an immunologist is over 10 years. The final analysis is expected to have over 1000 records and will relate complications to delay in diagnosis, treatment, and type of treatment.

Although all immunologists in this survey measured serum immunoglobulins, the number routinely carrying out further tests was below $60 \%$, despite evidence that significant immunodeficiency may occur in the presence of normal immunoglobulins. ${ }^{6}$ The consensus document indicates that it is appropriate for patients with suspected antibody deficiency to have lymphocyte surface markers, immunoglobulin G subclasses and specific antiviral and antibacterial antibodies measured, unless immunoglobulin concentrations are so low as to render the tests unnecessary. Thus the consensus standard was not being met in all centres.

The value of intramuscular replacement treatment for antibody deficiency was defined in an extensive Medical Research Council trial. ${ }^{7}$ From 1980 onwards safe and effective intravenous immunoglobulin became available and is now recognised as being superior to intramuscular immunoglobulin. ${ }^{8}$ It is a matter of concern that six immunologists would leave the choice of treatment to the patient. It is debatable in view of the evidence of efficacy of intravenous immunoglobulin whether it is acceptable practice to leave the patient, who relies on the specialist for the best possible advice, to choose between intramuscular and intravenous immunoglobulin. The choice of intramuscular immunoglobulin for patients with $\mathrm{X}$ linked agammaglobulinaemia is difficult to defend, as it is now clear that prompt introduction of intravenous immunoglobulin is essential to prevent the development of structural lung damage. ${ }^{910}$ The management of immunoglobulin $\mathrm{G}$ subclass deficiency and specific antibody deficiency is difficult, although some evidence is now emerging that intravenous immunoglobulin is desirable in treating these conditions and that continuous antibiotics are not as effective. ${ }^{6} 11$

Home treatment with intravenous immunoglobulin was introduced into the United Kingdom in $1986,{ }^{12}{ }^{13}$ and currently over 300 patients are established with home treatment. Despite the availability of this service eight immunologists did not offer this approach to treatment, thus disadvantaging their patients, even though home treatment results in greater participation of patients in their disease. ${ }^{5}$

For this group of rare diseases treatment is expensive: it is therefore important that it is used correctly. An objective monitor of the adequacy of treatment is trough immunoglobulin concentration. However, the optimum trough immunoglobulin $\mathrm{G}$ concentration is not known, and the only published report relates to the improvement in lung function seen if the trough concentration is $>5 \mathrm{~g} / 1 .^{9}$ Those immunologists setting targets for the trough immunoglobulin concentration below $5 \mathrm{~g} / \mathrm{l}$ are offering a treatment that is probably less than optimum: if they find that the outcome is satisfactory then the data need to be published and compared with existing data.

This survey has drawn attention to the difficulties of ensuring uniformity of treatment for rare diseases even between specialists. Although the approach taken by immunologists overall is generally uniform, there is a minority whose practice is at variance with their peers. The problems of poor quality care will be highlighted by the other part of this audit project which entails collecting data on morbidity and mortality in antibody deficiency. Dealing with the problem of inadequate care of patients by non-specialists will be difficult until the specialists themselves have ensured compliance with all the standards of the consensus document. We hope that the feedback of the survey data to the participants will highlight to individual immunologists where their practice differs from that of their peers. This survey is the first attempt at auditing the clinical performance of immunologists, but there is no formal mechanism and no regular national audit forum. The current project is funded only for a fixed period. The question of who sets standards of care for rare diseases and how they are monitored for appropriateness and for implementation has not been addressed in any systematic way but is worth study, as such diseases often involve expensive tertiary care.

By agreement, the consensus panel identified centres where it believes sufficient expertise exists, based on numbers of patients treated, to manage antibody deficiency effectively. ${ }^{5}$ However, this will not be effective in improving patient care unless purchasing authorities are prepared to take notice and direct their contracts accordingly. The NHS Executive has produced guidance on contracting for specialist services, ${ }^{14}$ but in many regions the recommendations are not fully implemented. Designation of specialist centres, with appropriate staffing and funding, is required to deal appropriately with rare and chronic diseases.

We thank all the immunologists who responded to the survey and were prepared to commit their practice to paper. This study forms part of a larger audit project, funded by the NHS Executive, and supported by the North of England Clinical Immunology Audit Group and the Association of Clinical Pathologists.

1 World Health Organisation. Primary immunodeficiency diseases. Report of a WHO sponsored meeting. Immunodefic Rev 1989;1:173-205.

2 Spickett GP, Misbah SA, Chapel HM. Primary antibody deficiency in adults. Lancet 1991;337:281-4

3 Björkander J, Bake B, Hanson LA. Primary hypogammaglobulinaemia: impaired lung function and body growth with delayed diagnosis and inadequate treatment. Eur $\mathcal{F}$ with delayed diagnosis and
Resp Dis 1984;65:529-36.

Resp Dis 1984;65:529-36.
4 Blore J, Haeney MR. Primary antibody deficiency and diagnostic delay. $B M \mathcal{F}$ 1989;298:516-7.

5 Chapel HM. Consensus on diagnosis and management of primary antibody deficiencies. $B M F$ 1994;308:581-5.

6 Knutsen AP. Patients with IgG subclass and/or selective antibody deficiency to polysaccharide antigens: initiation of a controlled clinical trial of intravenous immunoglobulin. $\mathcal{F}$ Allergy Clin Immunol 1989;84:640-7.

7 Medical Research Council Working Party on Hypogammaglobulinaemia. Hypogammaglobulinaemia in the United Kingdom. London: MRC-HMSO, 1971: vol SRS 310.

8 Sorensen RU, Polmar SH. Efficacy and safety of high dose intravenous immunoglobulin therapy for antibody deficiency syndromes. $A m \mathcal{F}$ Med 1984;(suppl):83-90. 
9 Roifman CM, Levison H, Gelfand EW. High-dose versus low-dose intravenous immunoglobulin in hypogammaglobulinaemia and chronic lung disease. Lancet 1987; 1075-7.

10 Liese JG Wintergerst U, Tympner KD, Belohradsky BH. High- vs low-dose immunoglobulin therapy in the longterm treatment of X-linked agammaglobulinemia. $\mathrm{Am} \mathrm{f}$ Dis Child 1992;146:335-9.

11 Silk HJ, Ambrosino D, Geha RS. Effect of intravenous gammaglobulin therapy in IgG2 deficient and IgG2 sufficient children with recurrent infections and poor response to immunisation with Hemophilus influenzae type b capsular polysaccharide. Ann Allergy 1990;64:21-5. 12 Chapel H, Brennan V, Delson E. Immunoglobulin replacement therapy by self infusion at home. Clin Exp Immunol 1988;73:160-2.

13 Ryan A, Thomson BJ, Webster ADB. Home intravenous immunoglobulin therapy for patients with primary hypoimmunoglobulin therapy for patients with primary hypo-

14 NHS Management Executive. Contracting for specialised services: a practical guide. Heywood: Health Publications Unit, 1993. 